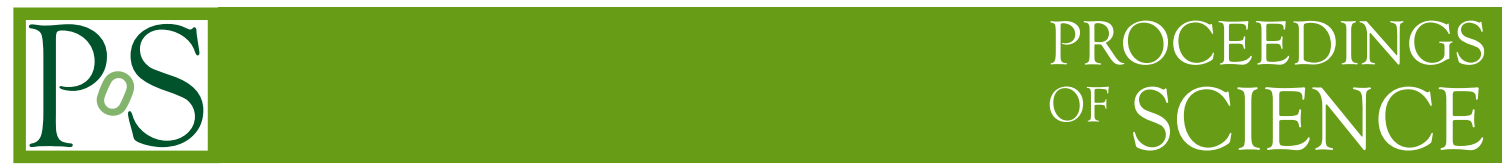

\title{
Diboson production at the LHC
}

\section{Lara Lloret Iglesias*}

Laboratorio de Instrumentacao e Fisica de Particulas (Lisbon)

Av. Elias Garcia 1411000 - 149 Lisbon (Portugal)

E-mail: lara@cern.ch

These proceedings present an overview of the diboson production cross-section measurements and constraints on anomalous triple-gauge boson couplings performed by the ATLAS and CMS collaborations using proton-proton collisions produced at a centre-of-mass energy of $\sqrt{s}=8$ and $13 \mathrm{TeV}$ at LHC. Results for all combinations of $\mathrm{W}, \mathrm{Z}$ and $\gamma$ gauge bosons are presented with emphasis on the new WZ production cross sections measured by ATLAS at $\sqrt{s}=13 \mathrm{TeV}$ and WW production cross section measured by CMS at $\sqrt{s}=13 \mathrm{TeV}$. New constraints on anomalous triple-gauge couplings have been set by both experiments at $8 \mathrm{TeV}$.

Fourth Annual Large Hadron Collider Physics

13-18 June 2016

Lund, Sweden

${ }^{*}$ Speaker. 


\section{Introduction}

In the Standard Model (SM) of particle physics, measurements of diboson final states at the $\mathrm{TeV}$ scale provide excellent tests of the electroweak sector. Any deviation of the diboson production cross sections or kinematic distributions from the SM predictions may be an indication of anomalous triple-gauge boson couplings (aTGC) and of the existence of new particles. It is therefore very important to have precise diboson measurements as well as reliable and accurate theoretical predictions for these processes. In addition non-resonant diboson production processes must be understood in detail as they represent a significant background to the measurements of the Higgs boson. The diboson results presented in these proceedings are based on the proton-proton collision data collected by the ATLAS and CMS detectors at the LHC.

\section{Diboson production cross-section measurements}

\section{1 $\mathrm{ZZ}$ production cross - section}

Studying the production of pairs of $\mathrm{Z}$ bosons in proton-proton (pp) interactions at the Large Hadron Collider (LHC) tests the electroweak sector of the standard model (SM) at the highest available energies. In pp collisions at a center-of-mass energy of $\sqrt{s}=13 \mathrm{TeV}, \mathrm{ZZ}$ production is dominated by quark-antiquark (qq) interactions, with an $\mathrm{O}(10 \%)$ contribution from loop-induced gluon-gluon (gg) interactions. The SM ZZ production can proceed via a Higgs boson propagator, although this contribution is suppressed in the region where both $\mathrm{Z}$ bosons are produced on-shell. As such, non-Higgs ZZ production is an important background in studies of the Higgs boson. It is also a background in searches for new physics producing pairs of $Z$ bosons at high invariant mass and sensitive to triple neutral gauge boson couplings, which are not allowed in the SM.

Candidate events are reconstructed in the fully leptonic $Z Z \rightarrow l^{+} l^{-} l^{+} l^{-}$decay channel where $l$ and $l^{\prime}$ can be an electron or a muon. The event selection requires 4 isolated leptons with the same flavour pairs having opposite charge. The luminosity used by ATLAS is $3.2 \mathrm{fb}^{-1}$ and by CMS 2.6 $\mathrm{fb}^{-1}$ being the on-shell $\mathrm{Z}$ mass selection $60-120 \mathrm{GeV}$ for CMS [1] and $66-116 \mathrm{GeV}$ for ATLAS [2] . For both experiments the cross section is measured in a fiducial phase space reflecting the detector acceptance. It is also extrapolated to the total phase space for $\mathrm{Z}$ bosons in the same mass range and of all decay modes. The major background contributions arise from $\mathrm{Z}$ boson and $\mathrm{WZ}$ diboson production in association with jets and from tt production. In all these cases, particles from jet fragmentation satisfy both lepton identification and isolation criteria, and are thus misidentified as signal leptons. The total expected number of final background events is extrapolated from specifically designated control regions. The systematic uncertainties are dominated by the statistics in the control sample used to estimate the backgrounds. The total cross section has been measured to be $\sigma(p p \rightarrow Z Z)=14.6_{-1.8}^{+1.9}(\text { stat })_{-0.3}^{+0.5}($ syst $) \pm 0.2$ (theo $) \pm 0.4$ (lumi) pb in CMS for Z bosons produced in the mass region $60<m_{Z}<120 \mathrm{GeV}$ to be compared with the NNLO prediction of $16.5_{-0.5}^{+0.7} \mathrm{pb}$ and $\sigma(p p \rightarrow Z Z)=16.7_{-2.0}^{+2.2}(\text { stat })_{-0.7}^{+0.9}(\text { syst })_{-0.7}^{+1.0}($ lumi $)$ in ATLAS for Z bosons produced in the mass region $66<m_{Z}<116 \mathrm{GeV}$ to be compared with the $O\left({ }_{s^{2}}\right)$ prediction $15.6_{-0.4}^{+0.4} \mathrm{pb}$. Both results are consistent with standard model predictions.

Figure 1 shows the distributions of the four-lepton invariant mass distribution and the total ZZ cross section measured by CMS as a function of the center of energy. Figure 2 shows the invariant 
mass of the leading versus the subleading lepton pair from ATLAS and the comparison between the measured fiducial cross sections and the theoretical predictions.
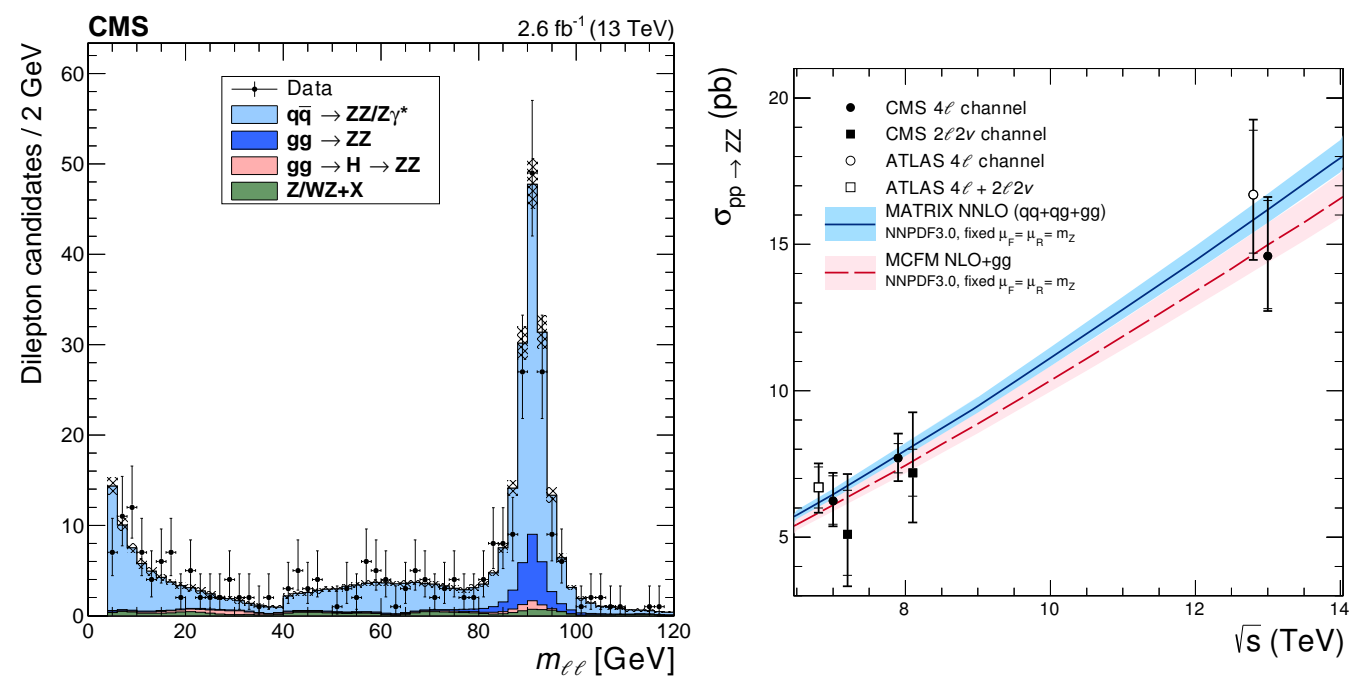

Figure 1: Distributions of the four-lepton invariant mass $m_{l^{+} l^{-} l^{+} l^{-}-}$with the CMS experiment (left) and the total $\mathrm{ZZ}$ cross section as a function of the proton-proton center-of-mass energy (right). Results from the CMS and ATLAS experiments are compared to predictions from MATRIX and MCFM with NNPDF3.0 PDF sets and fixed scales $\mu_{F}=\mu_{R}=m_{Z}$. Measurements at the same center-of-mass energy are shifted slightly along the $\mathrm{x}$-axis for clarity.
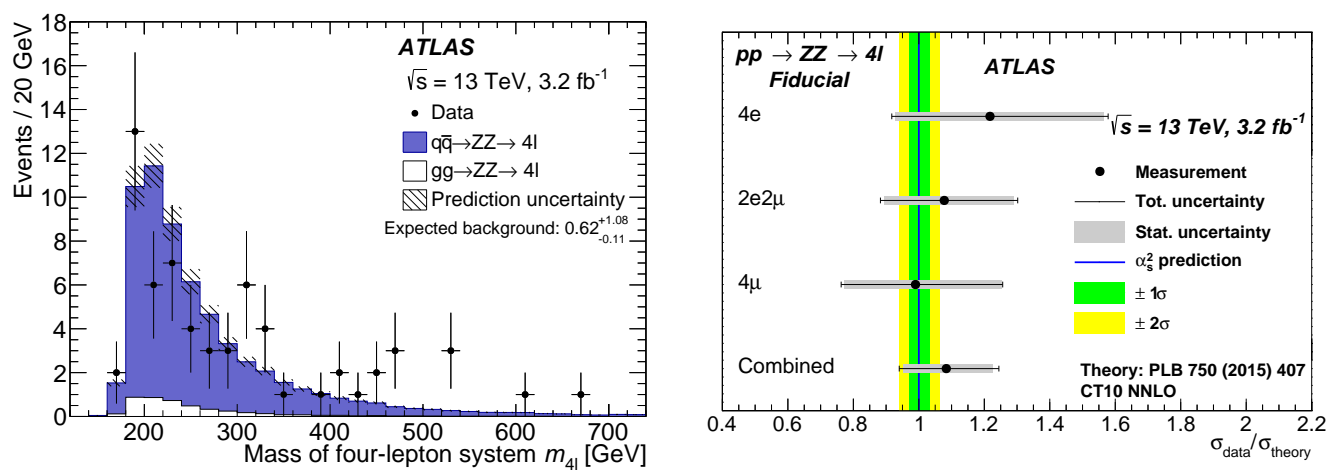

Figure 2: (left) Invariant mass of the four-lepton system in selected events. (right) Comparison between measured fiducial cross sections and $O\left({ }_{s^{2}}\right)$ predictions.

\subsection{WZ production cross-section}

The production of WZ pairs in hadron collisions is an important test of the electroweak sector of the Standard Model (SM). The WZ final states arise from two vector bosons radiated by quarks or from the decay of a virtual $\mathrm{W}$ boson into a WZ pair, which involves a triple gauge coupling (TGC). In addition, WZ pairs can be produced in vector-boson scattering processes, which involve triple and quartic gauge couplings (QGC) and are sensitive to the electroweak symmetry breaking sector of the SM. New physics could manifest in WZ final states as a modification of the TGC and 
QGC strength. Precise knowledge of the WZ production cross section is therefore necessary in the search for new physics.

WZ production cross section has been measured by the ATLAS detector [3] at $8 \mathrm{TeV}$ for a luminosity of $20.3 \mathrm{fb}^{-1}$. The total cross section at $8 \mathrm{TeV}$ was measured to be $\sigma_{W Z}=24.3 \pm$ 0.6 (stat. $) \pm 0.6$ (sys.) \pm 0.4 (th. $) \pm 0.5$ (lumi) pb to be compared with the NLO prediction of $21.0 \pm$ $1.6 \mathrm{pb}$.

The measurement has been performed by both CMS [4] and ATLAS [5] experiments at $13 \mathrm{TeV}$. In the case of CMS the collision events were selected by triggers that require the presence of one or two electrons or muons. The transverse momentum threshold for the single lepton trigger is 23 (20) $\mathrm{GeV}$ for the electron (muon) trigger. For the dilepton triggers, with the same or different flavors, the minimum $p_{T}$ of the leading and subleading leptons are 17 (17) and 12 (8) GeV for electrons (muons), respectively. The trigger efficiency for events within the acceptance of this analysis is greater than $99 \%$. A selected event is required to have three lepton candidates $l l^{\prime} l^{\prime}$. The $l^{\prime} l^{\prime}$ pair has two leptons with opposite charge and the same flavor, as expected for a $\mathrm{Z}$ boson candidate. One of the leptons from the $\mathrm{Z}$ boson candidate is required to have $p_{T}>20 \mathrm{GeV}$ and the other $p_{T}>10$ $\mathrm{GeV}$. If more than one combination is possible, the one with invariant mass closest to the $\mathrm{Z}$ boson mass is selected. The lepton associated with the $\mathrm{W}$ boson must have $p_{T}>20 \mathrm{GeV}$. All leptons must pass the tight identification and isolation requirements. To further reduce the contribution from $\mathrm{Z}+$ jets in events with an electron associated with the $\mathrm{W}$ boson, this electron must pass the requirements of the very tight working point. There must be no other isolated leptons with $p_{T}>10$ $\mathrm{GeV}$ in the events. To reduce contributions from tt events, the two leptons constituting the $\mathrm{Z}$ boson candidate are required to have an invariant mass satisfying $76<m_{l l}<106 \mathrm{GeV}$, and there must be no jets with $p_{T}>20 \mathrm{GeV}$ and $|\eta|<2.4$ that pass a $\mathrm{b}$ tagging requirement. The WZ events are expected to have missing transverse energy $\left(E_{T}^{\text {miss }}\right)$ consistent with the presence of a neutrino in the final state, therefore $E_{T}^{\text {miss }}>30 \mathrm{GeV}$ is required. The invariant mass of any dilepton pairs must be greater than $4 \mathrm{GeV}$. The trilepton invariant mass $\mathrm{m} 31$ is required to be more than $100 \mathrm{GeV}$.

The background contributions in this analysis are divided into two categories: background processes with prompt isolated leptons, e.g., ZZ,Z $\gamma$, ttZ; and background processes from nonprompt leptons from hadrons decaying to leptons inside jets or jets misidentified as isolated leptons, primarily $\mathrm{Z}+\mathrm{jets}$ and $\mathrm{tt}$. The background processes with prompt leptons are estimated from simulation. The processes with at least one nonprompt lepton are estimated from data. The major background contributions with nonprompt leptons arise from the production of $\mathrm{Z}$ bosons in association with jets and from tt, whereas smaller contributions come from $\mathrm{W}$ boson production in association with jets and multijet processes. The nonprompt background contribution is evaluated using the tightto-loose method. The method estimates the probability that a loose candidate is misidentified as a tight lepton and applies this probability to control regions with loose candidates to estimate the resulting contribution to the signal region. These loose candidates are selected with relaxed lepton identification and isolation requirements. The total $\mathrm{WZ}$ cross section measured at $13 \mathrm{TeV}$ by CMS using $2.3 \mathrm{fb}^{-1}$ is $\sigma(p p \rightarrow W Z)=40.9 \pm 3.4(\text { stat })_{-3.3}^{+3.1}($ syst $) \pm 0.4$ (theo $) \pm 1.3$ (lumi) pb for Z bosons produced in the mass region $60<m_{Z}<120 \mathrm{GeV}$ to be compared with the NLO prediction of $42.6_{-0.8}^{+1.6}$. Figure 3 shows the $l l^{\prime} l^{\prime}$ reconstructed mass for all decay channels and the transverse mass of the three leptons and the $E_{T}^{\text {miss }}$ system from the CMS analysis. 

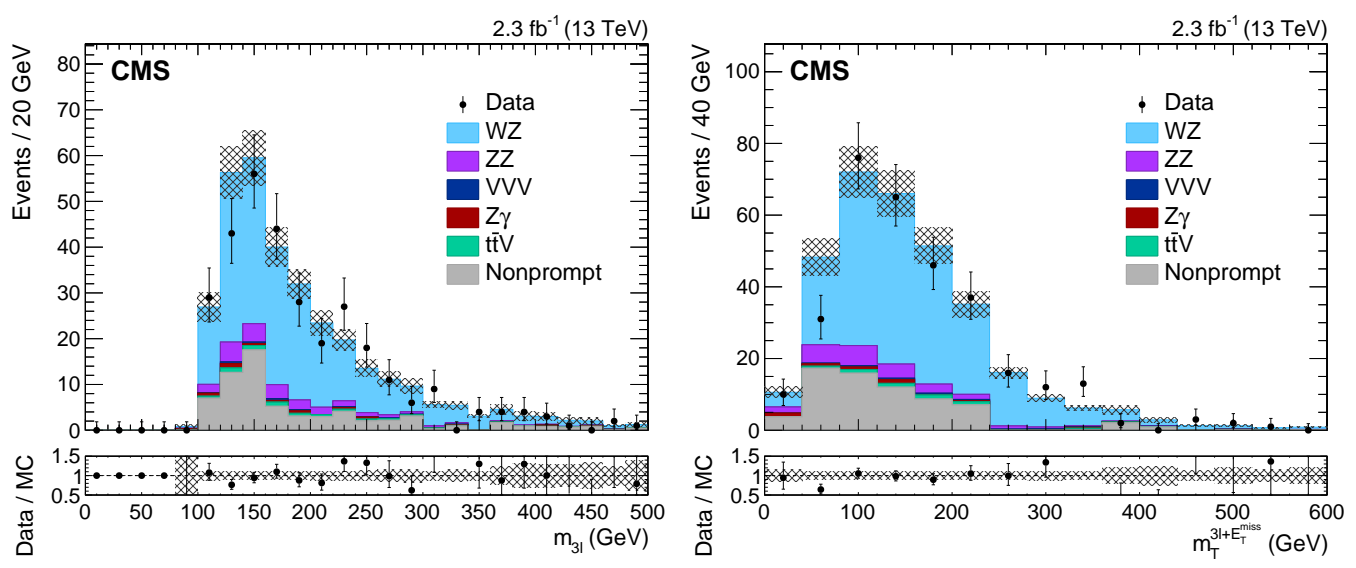

Figure 3: (left) Distribution of the $l l^{\prime} l^{\prime}$ reconstructed mass summed for all decay channels with the $m 3 l>100 \mathrm{GeV}$ selection requirement removed. (right) The transverse mass of the three leptons and the $E_{T}^{\text {miss }}$ system. Solid symbols represent the data with statistical uncertainty, while histograms represent the expected WZ signal and backgrounds. Uncertainties in the signal and background yields include experimental systematic, theoretical, and integrated luminosity uncertainties in addition to the statistical uncertainty. The background shapes are taken from simulation or data.

For the result by ATLAS candidate events are selected using triggers that require at least one electron or muon with $p_{T}>24 \mathrm{GeV}$ or $20 \mathrm{GeV}$, respectively, that satisfies a loose isolation requirement. Possible inefficiencies for 4 leptons with large transverse momenta are reduced by including additional electron and muon triggers that do not include any isolation requirements with transverse momentum thresholds of $p_{T}=60 \mathrm{GeV}$ and $50 \mathrm{GeV}$, respectively. Finally, a singleelectron trigger requiring $p_{T}>120 \mathrm{GeV}$ with less restrictive electron identification criteria is used to increase the selection efficiency for high- $p_{T}$ electrons. Events are required to have a primary vertex reconstructed from at least two charged particle tracks and compatible with the luminous region. If several such vertices are present in the event, the one with the highest sum of the $p_{T}$ of the associated tracks is selected as the primary vertex of the WZ production.

Events are required to contain exactly three lepton candidates satisfying the selection criteria described above. To ensure that the trigger efficiency is well determined, at least one of the candidate leptons is required to have $p_{T}>25 \mathrm{GeV}$ and to be geometrically matched to a lepton that was selected by the trigger. To suppress background processes with at least four prompt leptons, events with a fourth lepton candidate satisfying looser selection criteria are rejected. For this looser selection, the $p_{T}$ of the leptons is lowered to $7 \mathrm{GeV}$ and loose identification requirements are used for both the electrons and muons. The isolation requirement uses track information only and is less stringent. Candidate events are required to have at least one pair of leptons of the same flavour and of opposite charge, with an invariant mass that is consistent with the nominal $\mathrm{Z}$ boson mass to within $10 \mathrm{GeV}$. This pair is considered to be the $\mathrm{Z}$ boson candidate. If more than one pair can be formed, the pair whose invariant mass is closest to the nominal $\mathrm{Z}$ boson mass is taken as the $\mathrm{Z}$ boson candidate. The remaining third lepton is assigned to the $\mathrm{W}$ boson decay. The transverse mass of the $\mathrm{W}$ candidate, computed using $E_{T}^{\text {miss }}$ and the $p_{T}$ of the associated lepton, is required to be greater than $30 \mathrm{GeV}$. Backgrounds originating from misidentified leptons are suppressed by 
requiring the lepton associated with the $\mathrm{W}$ boson to satisfy more stringent selection criteria. Thus, the transverse momentum of these leptons is required to be greater than $20 \mathrm{GeV}$. Furthermore, electrons associated with the $\mathrm{W}$ boson decay are required to pass the tight likelihood identification requirement, which has an overall efficiency of $85 \%$. Finally, these electrons must also pass a tighter isolation requirement, tuned for an efficiency of at least $90 \%$ (99\%) for $p_{T}>25$ (60) $\mathrm{GeV}$. As in the CMS case the background sources are classified into two groups: events where at least one of the candidate leptons is not a prompt lepton (reducible background) and events where all candidates are prompt leptons or are produced in the decay of a $\tau$ (irreducible background). Candidates that are not prompt leptons are called also misidentified or fake leptons. The reducible background, which represents about half of the total backgrounds, originates from $Z+j, Z \gamma, \mathrm{tt}$, Wt and WW production processes, with $Z+j$ and $Z \gamma$ being the dominant component (83\%). The reducible backgrounds are estimated using data-driven techniques. The background from events with two or three fake leptons, e.g., from $\mathrm{W}+\mathrm{j} \mathrm{j}$ and multijet processes, is negligible. Irreducible background events originate from $\mathrm{ZZ}, \mathrm{tt} \mathrm{V}, \mathrm{VVV}$ (where $\mathrm{V}=\mathrm{Z}$ or $\mathrm{W}$ ), $\mathrm{tZ}$ and $\mathrm{WZ}$ events in which at least one of the bosons decays into leptons via an intermediate $\tau$ decay. The amount of irreducible background is estimated using MC simulations.

The total WZ cross section measured at $13 \mathrm{TeV}$ by ATLAS using $3.2 \mathrm{fb}^{-1}$ is measured to be $\sigma(p p \rightarrow W Z)=50.6 \pm 2.6($ stat $) \pm 2.0($ sys $) \pm 0.9($ th. $) \pm 1.2($ lumi $)$ pb to be compared with the NNLO prediction of $48.2_{-1.0}^{+1.1}$.

Both results, ATLAS and CMS, are in agreement with the expectations. Figure 4 shows the transverse mass variable Data/MC comparison and the ratio of the measured WZ integrated cross sections in the fiducial phase space in each of the four channels for ATLAS.

\subsection{WW production cross-section}

As the cross section for WW production is one of the largest among those involving a triplegauge boson vertex, it allows tests of the self-interaction of the gauge bosons to be made with high precision through measurements of differential kinematic distributions. Studies of the WW production process are particularly important as it constitutes a large irreducible background to searches for physics beyond the SM as well as to resonant $H \rightarrow W W$ production. A precise measurement of WW production also tests the validity of the theoretical calculations. The WW production cross section has been measured at $8 \mathrm{TeV}$ both by ATLAS [6] and CMS [7] detectors. For CMS the total WW cross section at $8 \mathrm{TeV}$ for a luminosity of $19.4 \mathrm{fb}^{-1}$ has been measured to be $\sigma(p p \rightarrow W W)=$ $60.1 \pm 0.9$ (stat) $\pm 3.2($ exp $) \pm 3.1($ th $) \pm 1.6$ (lumi) pb to be compared with the NNLO prediction of $59.8_{-1.1}^{+1.3} \mathrm{pb}$. For ATLAS the total WW cross section at $8 \mathrm{TeV}$ for a luminosity of $20.3 \mathrm{fb}^{-1}$ has been measured to be $\sigma(p p \rightarrow W W)=71.1 \pm 1.1(\text { stat })_{-5.0}^{+5.7}($ syst $) \pm 1.4($ lumi $)$ pb consistent with 1.4 standard deviations with respect to the NNLO prediction of $63.2_{-1.4}^{+1.6}$ (scale $) \pm 1.2(P D F) \mathrm{pb}$.

By the time of the conference the only measurement at $13 \mathrm{TeV}$ of the cross section of the WW process was performed by CMS [8]. The selection for this measurement consists of two oppositely charged, high $p_{T}$, isolated leptons not compatible with a $\mathrm{Z}$ boson decay, large missing energy from the neutrinos recoiling against the two leptons, and a small number of jets. Selected events contain exactly one electron and one muon with opposite charge and $p_{T}>20 \mathrm{GeV}$, which greatly suppresses contributions from Drell Yan (DY) processes. Events containing a loosely identified third lepton with $p_{T}>10$ are rejected to supress backgrounds from $\mathrm{WZ}$ and $\mathrm{ZZ}$ processes. The missing 

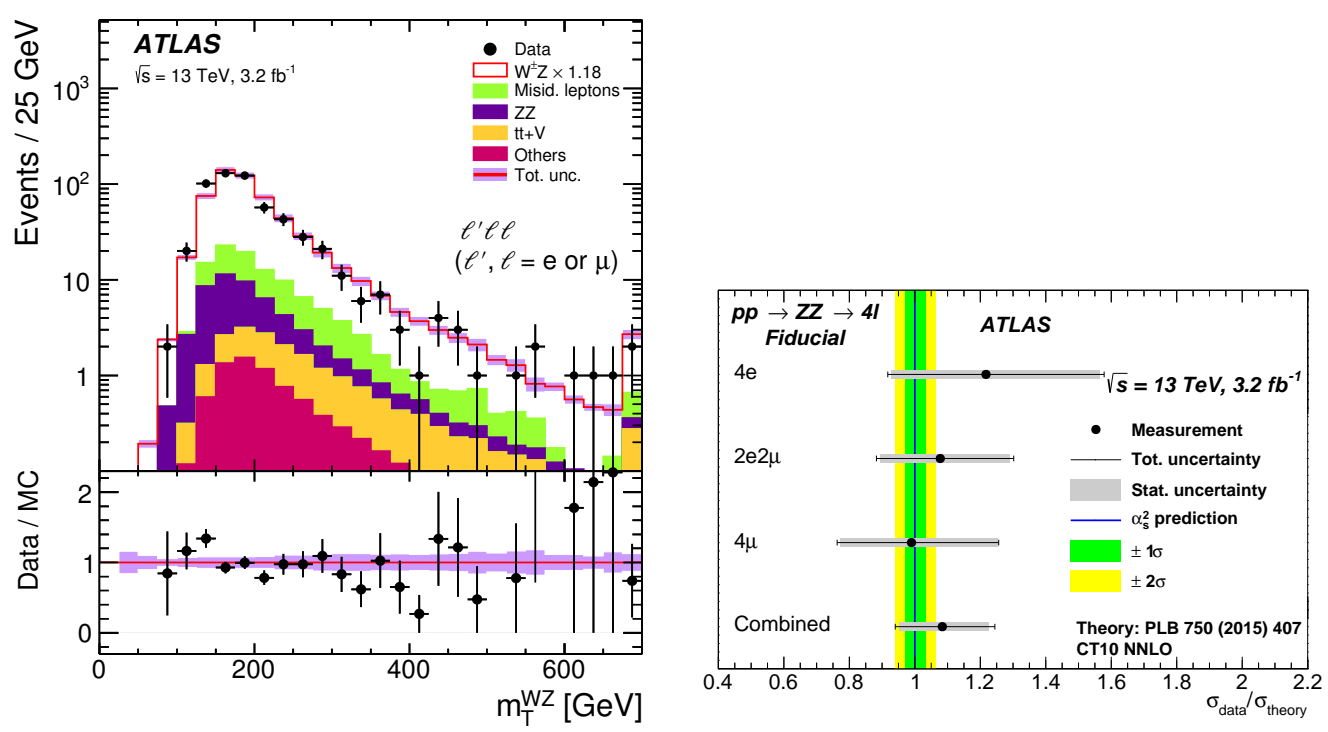

Figure 4: (left) The transverse mass variable $m_{T}^{W Z}$ for the WZ system. The points correspond to the data, and the histograms correspond to the predictions of the different SM processes. All Monte Carlo predictions are scaled to the integrated luminosity of the data using the predicted MC cross sections of each sample. The sum of the background processes with misidentified leptons is labelled misidentified leptons. The Powheg+Pythia MC prediction is used for the WZ signal contribution. It is scaled by a global factor of 1.18 to match the measured inclusive WZ cross section. The open red histogram shows the total prediction; the shaded violet band is the total uncertainty of this prediction. The last bin contains the overflow. The lower panel in each figure show the ratio of the data points to the open red histogram with their respective uncertainties. (right) Ratio of the measured WZ integrated cross sections in the fiducial phase space to the NLO SM prediction from Powheg+Pythia in each of the four channels and for their combination. The inner and outer error bars on the data points represent the statistical and total uncertainties, respectively. The NLO SM prediction from Powheg+Pythia using the CT10 PDF set is represented by the red line; the shaded violet band is the total uncertainty in this prediction.

transverse energy in the events is required to be larger than $20 \mathrm{GeV}$. In order to make the analysis insensitive to instrumental $E_{T}^{\text {miss }}$ from mismeasurements of the lepton momenta, when the azimuthal angle, measured in a plane perpendicular to the beam axis, between the $E_{T}^{\text {miss }}$ vector and the closest lepton direction is smaller than $\pi / 2$, the component of $E_{T}^{\text {miss }}$ perpedicular to the transverse momentum of the closest lepton is required to be larger than $20 \mathrm{GeV}$. The same requirement is imposed using the $E_{T}^{\text {miss }}$ vector reconstructed from only the charged particle-flow candidates associated to the primary vertex. Only events with zero or one reconstructed jets with $p_{T}^{j}>30 \mathrm{GeV}$ and $\left|\eta_{j}\right|<$ 4.7 are used in the analysis, which supresses contributions from top-quark mediated processes. The measurement is performed separately for events with zero and one reconstructed jets. In order to further suppress top-quark events, events with loosely identified $\mathrm{b}$-quark jets with $p_{T}^{b}>20 \mathrm{GeV}$ are rejected. Finally, the transverse momentum and the invariant mass of the $e \mu$ system are required to be greater than $30 \mathrm{GeV}$ and $12 \mathrm{GeV}$, respectively. The former condition on the dilepton system suppresses backgrounds from misidentified jets while the second supresses the contribution from low-mass DY processes. A combination of data-driven methods and detailed simulated studies are used to estimate background contributions. The non-prompt lepton background, originating 
from leptonic decays of heavy quarks, hadrons misidentified as leptons, and electrons from photon conversions in $\mathrm{W}+$ jets and QCD multijet production, is suppressed by the identification and isolation requirements on electrons and muons. The remaining contribution from the non-prompt lepton background is estimated from data using the tight to loose method. The background from top-quark mediated processes is supressed by rejecting events with soft $b$-quark jets. The reduction of the top-quark background is about $47 \%$ in the 0 -jet category and about $83 \%$ in the 1 -jet category. The remaining top-quark background that passes the veto is estimated in a top-quark enriched control region and then extrapolated to the signal region. Background events from $\mathrm{W} \gamma^{*}$ processes with low mass $\gamma^{*}$ fulfill the event selection when the final state is highly asymmetric in transverse momentum. This component of the background is normalized, for $m_{\gamma^{*}}<4 \mathrm{GeV}$, in a control region with three muons satisfying $p_{T}>10,5$, and $3 \mathrm{GeV}$, for the leading, subleading, and trailing muon, respectively. In this control region, the missing transverse energy is required to satisfy $E_{T}^{\text {miss }}<25$ $\mathrm{GeV}$ in order to supress other sources of background events. All other background processes are estimated using simulated events, where each process is normalized to the most precise prediction for their cross section available.

CMS has measured the total cross section of the WW process at $13 \mathrm{TeV}$ for a luminosity of 2.3 $\mathrm{fb}^{-1}$ to be $\sigma(p p \rightarrow W W)=115.3 \pm 5.8$ (stat) \pm 5.7 (exp) \pm 6.4 (theo) \pm 3.6 (lum) pb to be compared with the NNLO prediction of $120.3 \pm 3.6 \mathrm{pb}$.

Figure 5 shows the comparison data/MC for the leading lepton and for the $E_{T}^{\text {miss }}$.
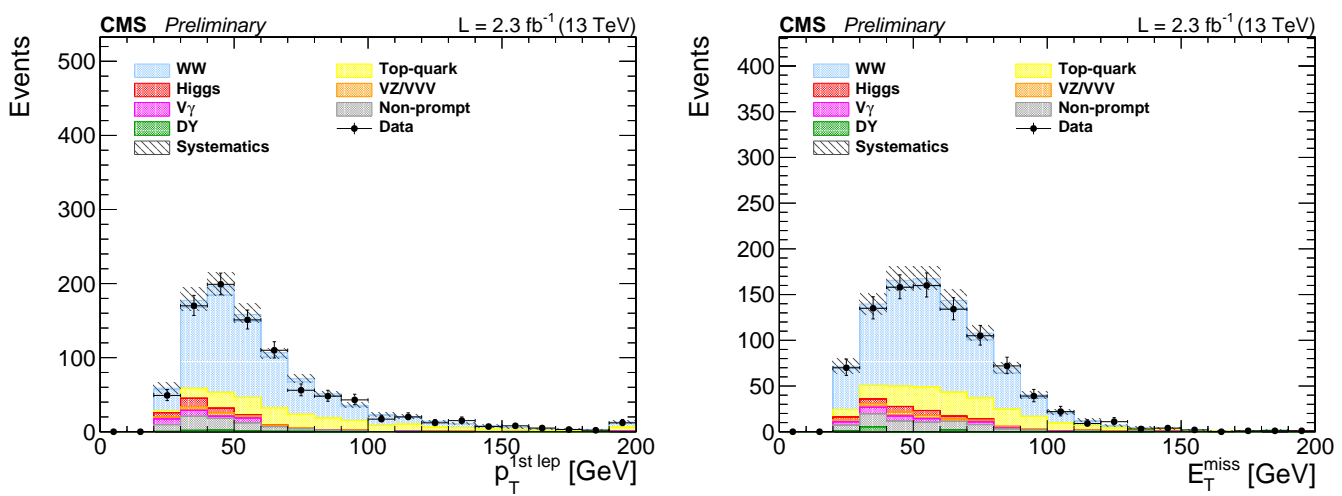

Figure 5: Comparison between data and $\mathrm{MC}$ distributions for the leading lepton $p_{T}$ (left) and for the missing transverse energy $E_{T}^{\text {miss }}$ (right) in the 0 -jet category. The hatched areas represent the systematic uncertainty in each bin. The last bin includes the overflow.

\section{Summary tables}

This section shows the summary tables [9][10] with all the results for the diboson cross section measurements for ATLAS (figure 6) and CMS (figure 7).

\section{Anomalous Gauge Couplings}

Anomalous couplings result in an increase of diboson cross section at high energies. Observables particularly sensitive to these couplings are proportional to the invariant mass of the diboson 


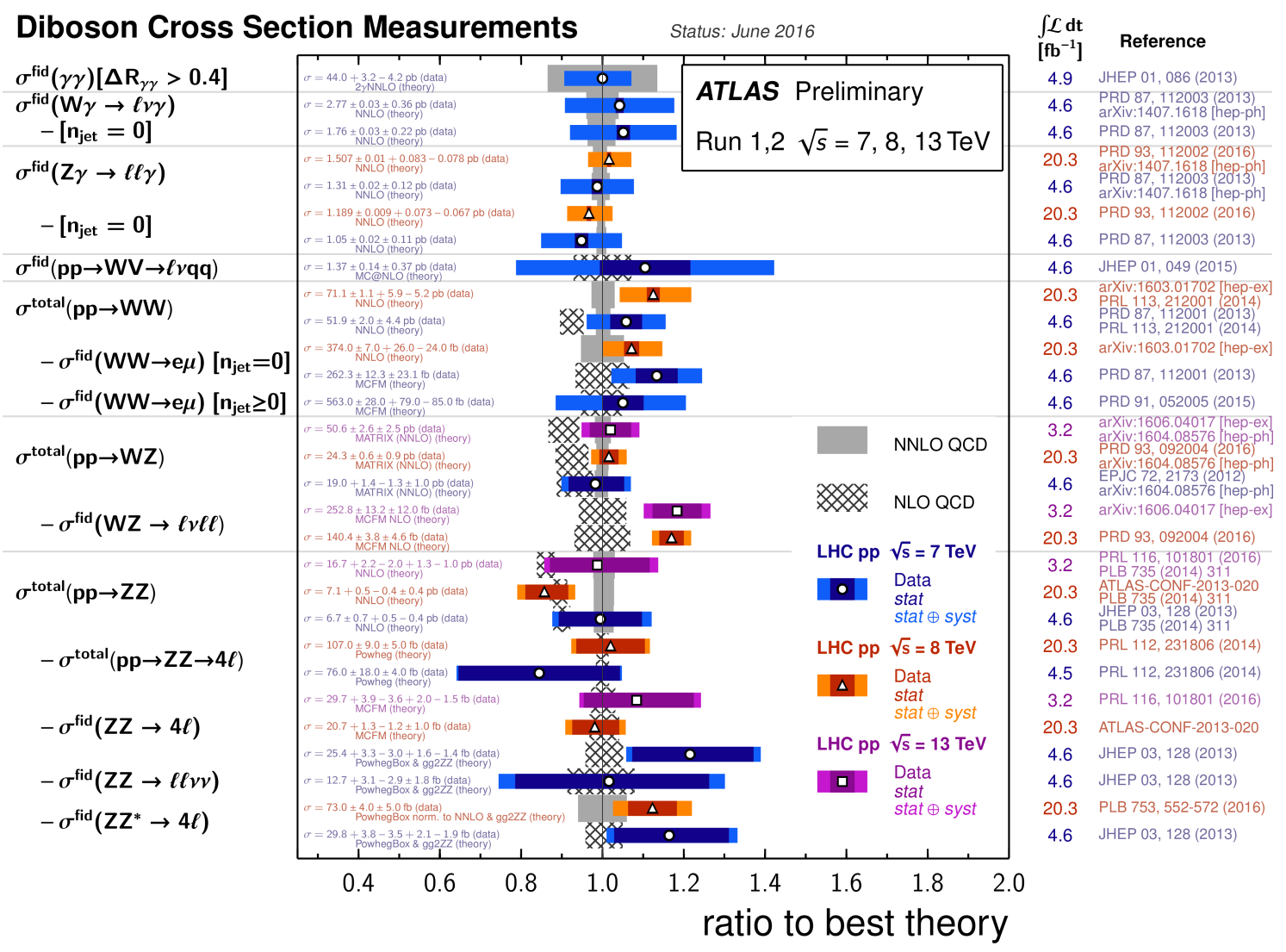

Figure 6: The ratio for several diboson total and fiducial production cross section measurements over best available theory prediction, corrected for leptonic branching fractions. All theoretical expectations are shown using gray bars, hatched for NLO calculations and full for NNLO predictions. All measurements were performed using the 2011 dataset, the 2012 dataset, or the 2015 dataset. The dark-color error bar represents the statistical uncertainly. The lighter-color error bar represents the full uncertainty, including systematics and luminosity uncertainties. The luminosity used and reference for each measurement are also shown. Uncertainties for the theoretical predictions are quoted from the original ATLAS papers. They were not always evaluated using the same prescriptions for PDFs and scales. The $\mathrm{W} \gamma$ and $\mathrm{Z} \gamma$ theoretical crosssections have non-perturbative corrections applied to the NNLO fixed order calculations (PRD 87, 112003 (2013)).

system or the boson $p_{T}$. One of the limiting factors in extracting the limits are the observed statistics in the tail (primary) and the systematic and statistical uncertainty on the signal model (secondary). The sensitivity depends on the absolute size of the anomalous coupling signal, absolute size of expected background and the different uncertainties. The different analyses are optimized to reach highest expected sensitivity. An effective lagrangian featuring such anomalous triple-gauge boson couplings (aTGCs) can be constructed and then compared to the experimental data. It can be parametrised by the parameters shown in table 1 . All these parameters are equal to zero in the SM. A summary with the current status on the limits set by ATLAS, CMS, LEP and Tevatron [11] is shown in figures [8-11]. No deviations from the SM are observed. 


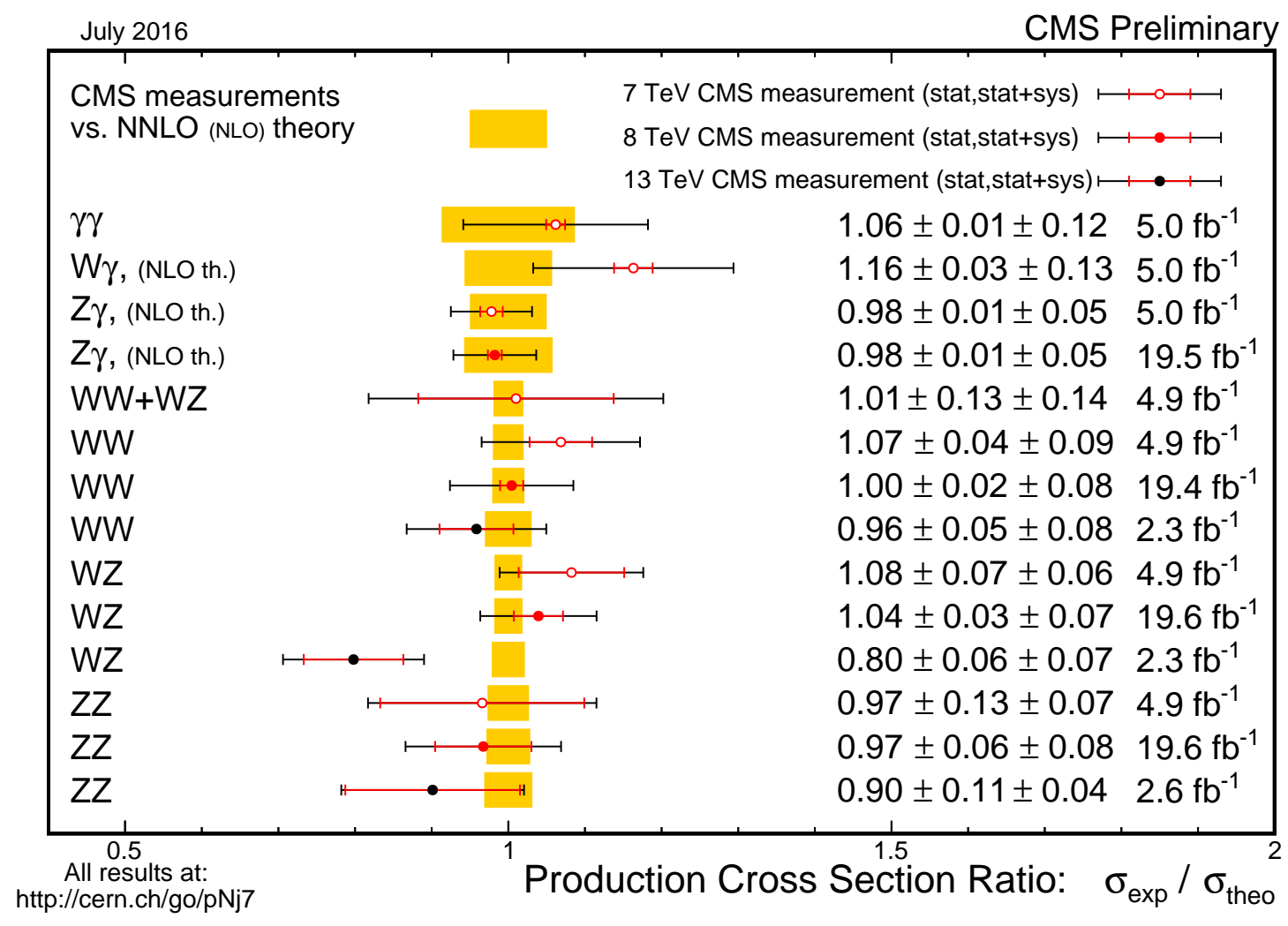

Figure 7: Di-boson cross section ratio comparison to theory: Theory predictions updated to latest NNLO calculations where available compared to predictions in the CMS papers and preliminary physics analysis summaries.

\begin{tabular}{|c|c|}
\hline Charged TGC & Neutral TGC \\
\hline$\Delta \kappa_{Z}, \lambda_{Z}, \Delta g_{1}^{Z}, \Delta \kappa_{\gamma}, \lambda_{\gamma}$ & $f_{4}^{\gamma}, f_{4}^{Z}, f_{5}^{\gamma}, f_{5}^{Z}, h_{3}^{\gamma}, h_{3}^{Z}, h_{4}^{\gamma}, h_{4}^{Z}$ \\
\hline
\end{tabular}

Table 1: Anomalous coupling parameters.

\section{Conclusions}

The LHC has shown a very good performance and has provided good data for both Run I and Run II. Several full analysis results from Run I at $8 \mathrm{TeV}$ have been published and some diboson cross sections have already been measured in Run II at $13 \mathrm{TeV}$. Differences have been observed when comparing with NLO predictions but a good agreement when comparing with NNLO predictions is found. Anomalous couplings searches show no deviation from the Standard Model prediction.

\section{References}

[1] CMS Collaboration. Measurement of the ZZ production cross section and Z to $l^{+} l^{-} l^{\prime} l^{\prime}$ branching fraction in pp collisions at $\sqrt{s}=13 \mathrm{TeV}$. arXiv preprint arXiv:1607.08834, 2016.

[2] ATLAS Collaboration. Measurement of the ZZ Production Cross Section in pp Collisions at $\sqrt{s}=13$ TeV with the ATLAS Detector. Physical review letters, 2016, vol. 116, no 10, p. 101801. 


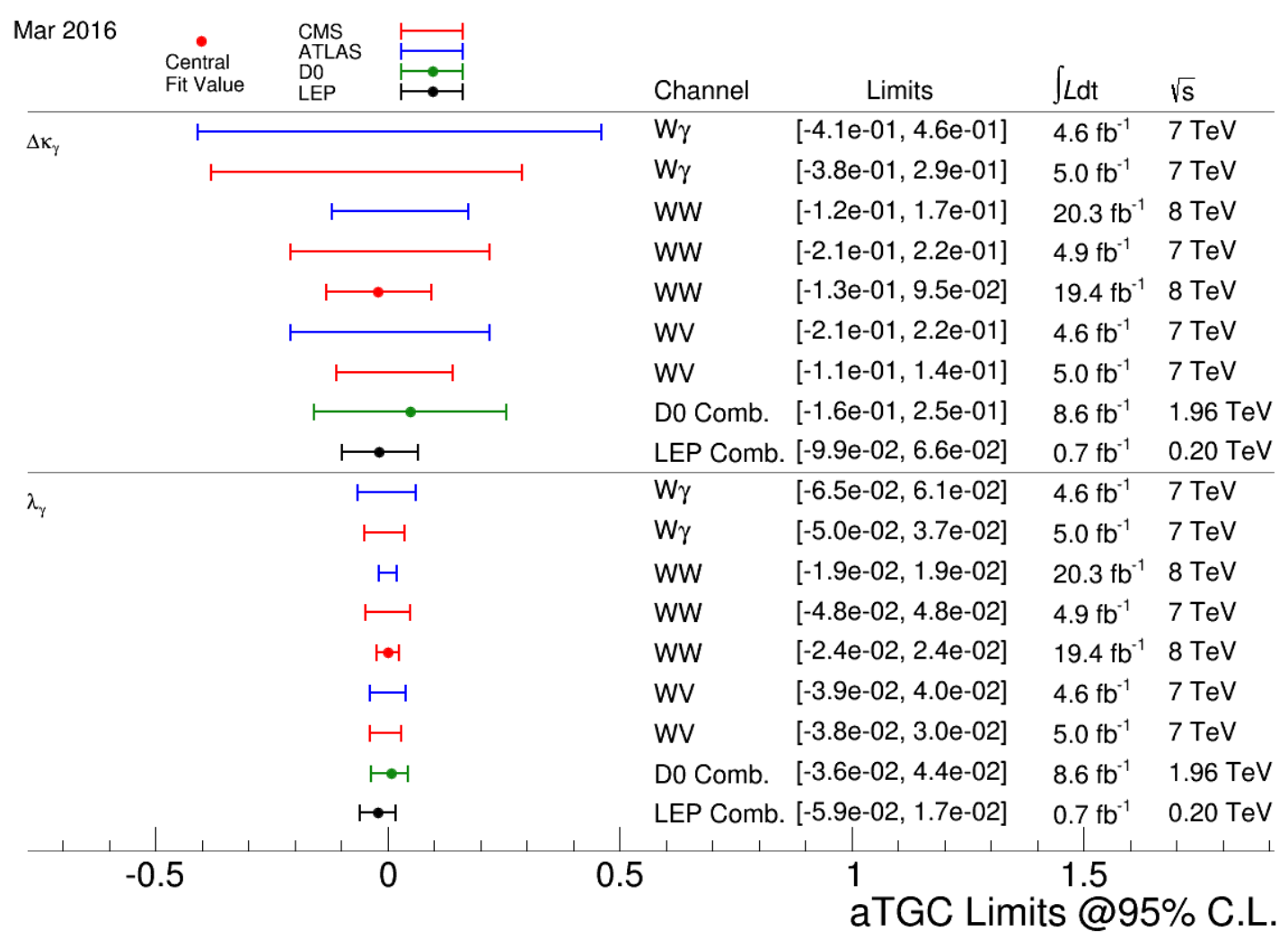

Figure 8: Limits on WW $\gamma$ aTGC.

[3] ATLAS Collaboration. Measurements of WZ production cross sections in p p collisions at $\sqrt{s}=8$ $\mathrm{TeV}$ with the ATLAS detector and limits on anomalous gauge boson self-couplings. Physical Review D, 2016, vol. 93, no 9, p. 092004.

[4] CMS Collaboration. Measurement of the WZ production cross section in pp collisions at $\sqrt{s}=13$ TeV. arXiv preprint arXiv:1607.06943, 2016.

[5] ATLAS Collaboration. Measurement of the WZ production cross section in pp collisions at $\sqrt{s}=13$ TeV arXiv preprint arXiv:1606.04017, 2016.

[6] ATLAS Collaboration. Measurement of total and differential WW production cross sections in proton-proton collisions at $\sqrt{s}=8 \mathrm{TeV}$ with the ATLAS detector and limits on anomalous triple-gauge-boson couplings. arXiv preprint arXiv:1603.01702, 2016.

[7] CMS Collaboration. Measurement of the WW cross section in pp collisions at $\sqrt{s}=8 \mathrm{TeV}$ and limits on anomalous gauge couplings. arXiv preprint arXiv:1507.03268, 2015.

[8] CMS Collaboration. Measurement of the WW cross section in pp collisions at $\sqrt{s}=13 \mathrm{TeV}$. CMS-PAS-SMP-16-006.

[9] https://twiki.cern.ch/twiki/bin/view/AtlasPublic/StandardModelPublicResults

[10] https://twiki.cern.ch/twiki/bin/view/CMSPublic/PhysicsResultsCombined

[11] https://twiki.cern.ch/twiki/bin/view/CMSPublic/PhysicsResultsSMPaTGC 


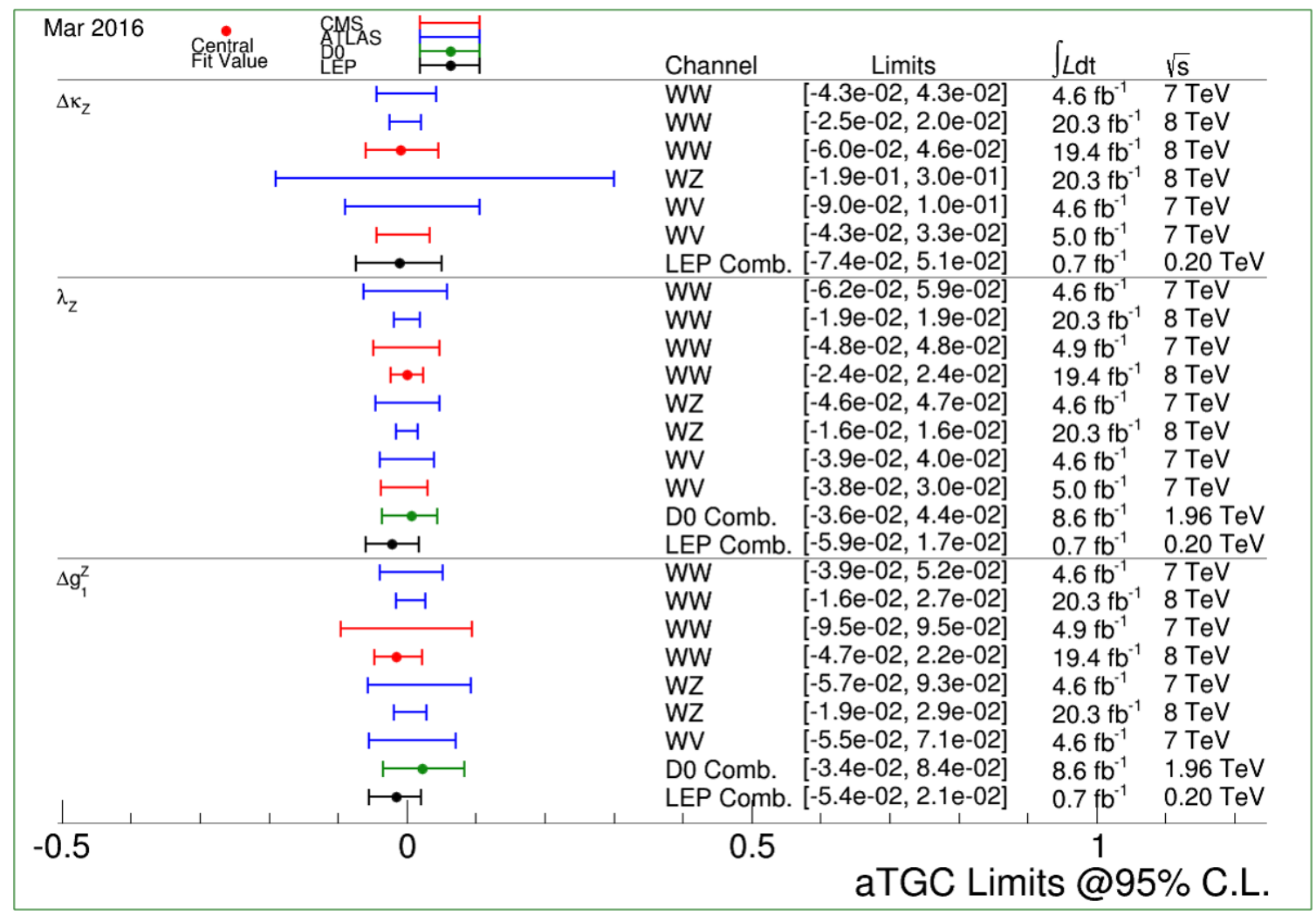

Figure 9: Limits on WWZ aTGC. 


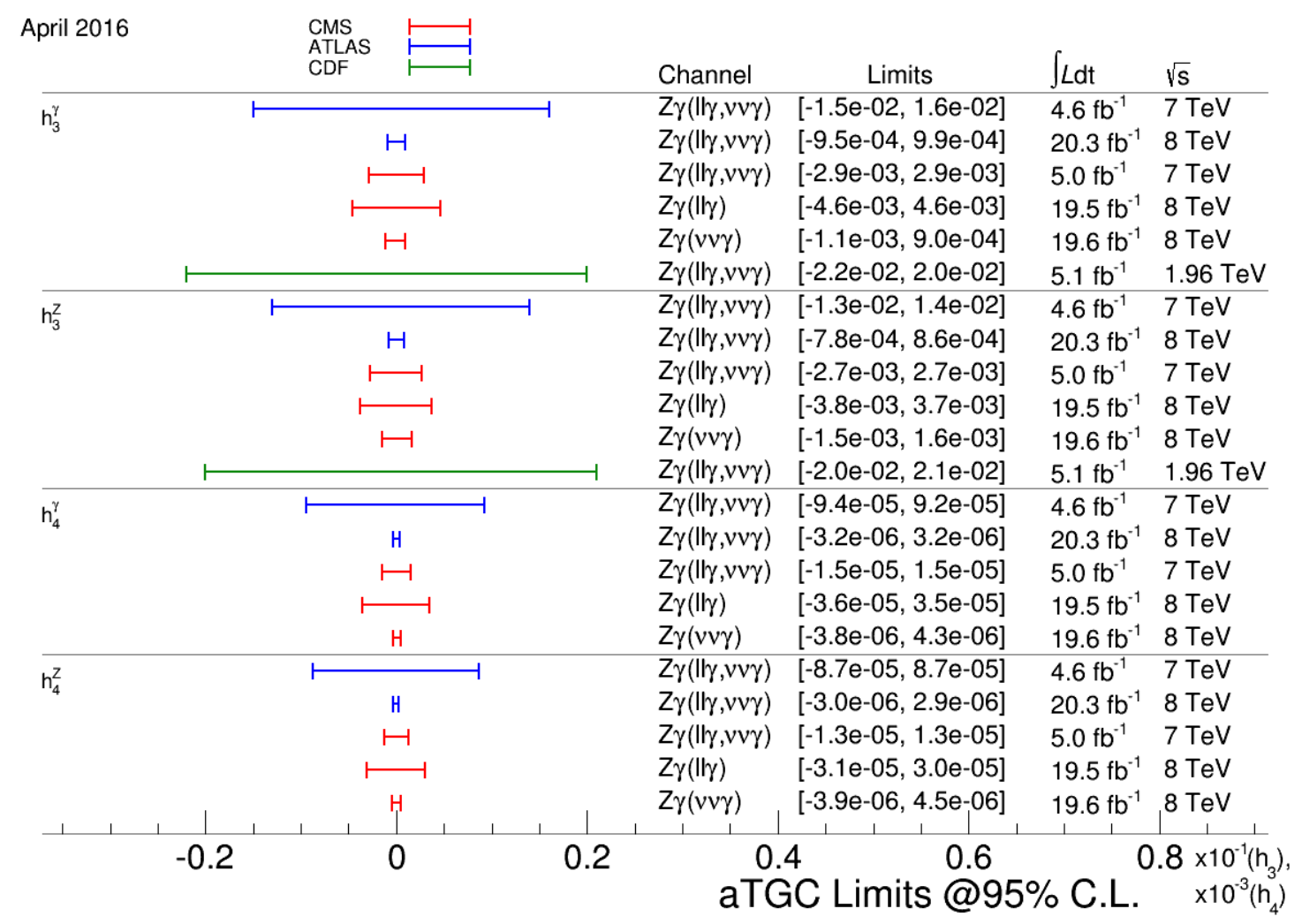

Figure 10: Limits on neutral aTGC $Z \gamma \gamma$ and $Z Z \gamma$. 


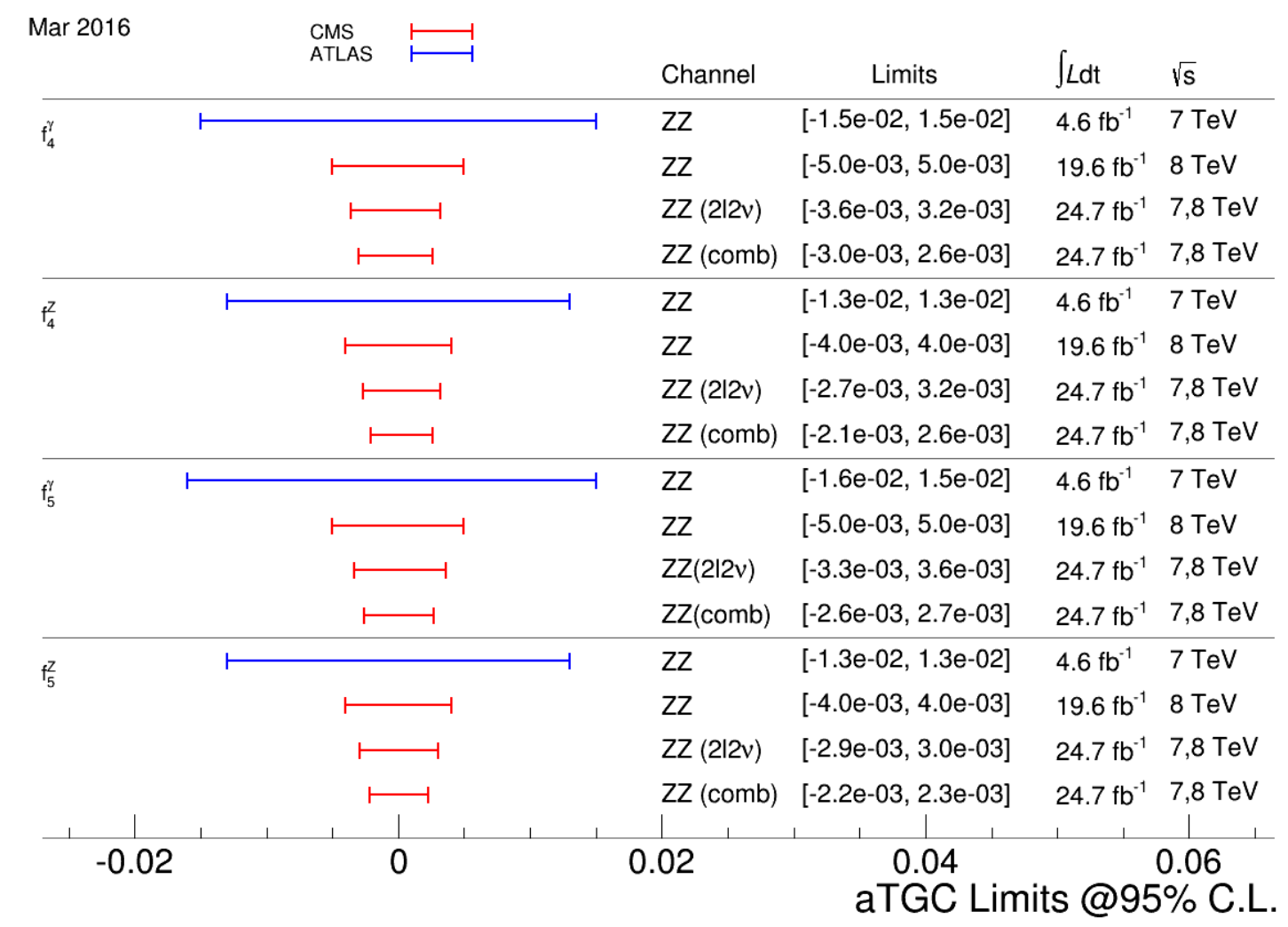

Figure 11: Limits on neutral aTGC. 